\title{
SMASH (CO)PRODUCTS AND SKEW PAIRINGS
}

\author{
J. N. Alonso Alvarez, J. M. Fernández Vilaboa and \\ R. GonzÁlez Rodríguez
}

\begin{abstract}
Let $\tau$ be an invertible skew pairing on $(B, H)$, where $B$ and $H$ are Hopf algebras in a symmetric monoidal category $\mathcal{C}$ with (co)equalizers. Assume that $H$ is quasitriangular. Then we obtain a new algebra structure such that $B$ is a Hopf algebra in the braided category ${ }_{H}^{H} \mathcal{Y D}$ and there exists a Hopf algebra isomorphism $w: B \infty H \rightarrow B \bowtie_{\tau} H$ in $\mathcal{C}$, where $B \infty H$ is a Hopf algebra with (co)algebra structure the smash (co)product and $B \bowtie_{\tau} H$ is the Hopf algebra defined by Doi and Takeuchi.
\end{abstract}

\section{Introduction}

The smash product algebra and the smash coproduct coalgebra are well know in the theory of Hopf algebras. If $B$ and $H$ are Hopf algebras Radford [10] found necessary and sufficient conditions for the smash product algebra structure and the smash coproduct coalgebra structure on $B \otimes H$ to afford $B \otimes H$ a Hopf algebra structure. Also, in [10], Radford describes completely the structure of Hopf algebras with a projection. Assume $B$ and $H$ Hopf algebras and $j: B \rightarrow H$ anf $f: H \rightarrow B$ Hopf algebra morphisms such that $j \circ f=\operatorname{id}_{H}$. Then, $B$ decomposes as a tensor product $D \infty H$ where $D$ is a Hopf algebra in the braided monoidal category of Yetter-Drinfeld modules and $\infty$ denotes the smash (co)product. Afterwards, Majid in [8] and Bespalov in [2] obtain a braided interpretation of Radford's theorem. In [1] this result is proved in the context of braided categories, using the notions of $H$-Cleft comodule (module) algebras (coalgebras). On the other hand, Doi and Takeuchi in [6] studied the double crossproducts $B \bowtie_{\tau} H$. These are determined by an skew pairing $\tau: B \otimes H \rightarrow K$ where $B$ and $H$ bialgebras. When $B$ and $H$ are Hopf algebras and $\tau$ is a convolution invertible skew pairing, $\left(B, \varphi_{B}=\right.$

2000 Mathematics Subject Classification. 16W30, 17B37, 18D10, 81R50.

Key words. Quasitriangular Hopf algebra, monoidal category, skew pairing, smash (co)product. 
$\left.\left(\tau \otimes B \otimes \tau^{-1}\right) \circ\left(B \otimes H \otimes \delta_{B} \otimes H\right) \circ \delta_{B \otimes H} \circ c_{H, B}\right)$ is a left $H$-module coalgebra and $\left(H, \psi_{H}=\left(\left(\tau \circ c_{H, B}\right) \otimes H \otimes\left(\tau^{-1} \circ c_{H, B}\right)\right) \circ\left(H \otimes B \otimes \delta_{H} \otimes B\right) \circ \delta_{H \otimes B}\right)$ is a right $B$-module coalgebra. The construction of $B \bowtie_{\tau} H$ is an example of Majid's double crossproduct $B \bowtie H[\mathbf{9}]$ because the Majid double crossproduct is defined as coalgebra $B \bowtie H=B \otimes H$ and the product is equal to the one defined in [6] for $B \bowtie_{\tau} H$. In [6], it is shown that, if $H$ is quasitriangular, there exists a Hopf algebra projection $g: B \bowtie_{\tau} H \rightarrow H$ and therefore, using the Radford's theorem, it is possible to find $D \in{ }_{H}^{H} \mathcal{Y D}$ such that $D \propto H$ is isomorphic with $B \bowtie_{\tau} H$. In this paper we prove that the Hopf algebra $B \bowtie_{\tau} H$, defined by Doi and Takeuchi, is an $H$-Cleft comodule algebra and an $H$-Cleft module coalgebra and then using Theorem 3.2 of [1] we show that the object $D$ such that $D \propto H \approx B \bowtie_{\tau} H$ is $B$ with a modified Hopf algebra structure. As a consequence, we obtain that $\left(B, \varphi_{B}\right)$ is an $H$-module algebra too. Analogously, we have a similar result for the dual case studied by Caenepeel, Dăscălescu, Militaru and Panaite in [3]. The proof can be derived easily from the one developed in Theorem 4.1.

\section{Preliminaries}

We assume the reader is familiar with the machinery of monoidal categories. Details may be found in [7]. By $(\mathcal{C}, \otimes, c, K)$ we denote a strict symmetric monoidal category with (co)equalizers where $c$ is the natural isomorphism of symmetry and $K$ is the base object. For every object $A$ in $\mathcal{C}, \operatorname{id}_{A}$ denotes the identity morphism.

An algebra in $\mathcal{C}$ is a triple $A=\left(A, \eta_{A}, \mu_{A}\right)$ where $A$ is an object in $\mathcal{C}$ and $\eta_{A}: K \rightarrow A, \mu_{A}: A \otimes A \rightarrow A$ are morphisms in $\mathcal{C}$ such that $\mu_{A} \circ\left(A \otimes \eta_{A}\right)=\operatorname{id}_{A}=\mu_{A} \circ\left(\eta_{A} \otimes A\right), \mu_{A} \circ\left(A \otimes \mu_{A}\right)=\mu_{A} \circ\left(\mu_{A} \otimes A\right)$. Given two algebras $A=\left(A, \eta_{A}, \mu_{A}\right)$ and $B=\left(B, \eta_{B}, \mu_{B}\right), f: A \rightarrow B$ is an algebra morphism if $\mu_{B} \circ(f \otimes f)=f \circ \mu_{A}, f \circ \eta_{A}=\eta_{B}$. Also, if $A, B$ are algebras in $\mathcal{C}$, the product algebra is $A B=\left(A \otimes B, \eta_{A \otimes B}=\right.$ $\left.\eta_{A} \otimes \eta_{B}, \mu_{A \otimes B}=\left(\mu_{A} \otimes \mu_{B}\right) \circ\left(A \otimes c_{B, A} \otimes B\right)\right)$.

A coalgebra in $\mathcal{C}$ is a triple $D=\left(D, \varepsilon_{D}, \delta_{D}\right)$ where $D$ is an object in $\mathcal{C}$ and $\varepsilon_{D}: D \rightarrow K, \delta_{D}: D \rightarrow D \otimes D$ are morphisms in $\mathcal{C}$ such that $\left(\varepsilon_{D} \otimes D\right) \circ \delta_{D}=\operatorname{id}_{D}=\left(D \otimes \varepsilon_{D}\right) \circ \delta_{D},\left(\delta_{D} \otimes D\right) \circ \delta_{D}=\left(D \otimes \delta_{D}\right) \circ \delta_{D}$. If $D=\left(D, \varepsilon_{D}, \delta_{D}\right)$ and $E=\left(E, \varepsilon_{E}, \delta_{E}\right)$ are coalgebras, $f: D \rightarrow E$ is a coalgebra morphism if $(f \otimes f) \circ \delta_{D}=\delta_{E} \circ f, \varepsilon_{E} \circ f=\varepsilon_{D}$. When $D$, $E$ are coalgebras in $\mathcal{C}$, the product coalgebra is $D E=\left(D \otimes E, \varepsilon_{D \otimes E}=\right.$ $\left.\varepsilon_{D} \otimes \varepsilon_{E}, \delta_{D \otimes E}=\left(D \otimes c_{D, E} \otimes E\right) \circ\left(\delta_{D} \otimes \delta_{E}\right)\right)$.

Let $D$ be a coalgebra and let $A$ be an algebra. By $\operatorname{Reg}(D, A)$ we denote the set of invertible morphisms $f: D \rightarrow A$ in $\mathcal{C}$ respect to the 
convolution operation $f * g=\mu_{A} \circ(f \otimes g) \circ \delta_{D} \cdot \operatorname{Reg}(D, A)$ is a monoid where the unit element is $\varepsilon_{D} \otimes \eta_{A}$.

Let $A$ be an algebra. $\left(M, \psi_{M}\right)$ is a right $A$-module if $M$ is an object in $(\mathcal{C}, c)$ and $\psi_{M}: M \otimes A \rightarrow M$ is a morphism in $\mathcal{C}$ satisfying $\psi_{M} \circ\left(M \otimes \eta_{A}\right)=$ $\operatorname{id}_{M}, \psi_{M} \circ\left(\psi_{M} \otimes A\right)=\psi_{M} \circ\left(M \otimes \mu_{A}\right)$. Given two right $A$-modules $\left(M, \psi_{M}\right)$ and $\left(N, \psi_{N}\right), f: M \rightarrow N$ is a morphism of right $A$-modules if $\psi_{N} \circ(f \otimes A)=f \circ \psi_{M}$. We denote the category of right $A$-modules by $\mathcal{C}_{A}$. In an analogous way we define the left $A$-modules and we denote this category by ${ }_{A} \mathcal{C}$.

Let $D$ be a coalgebra. $\left(M, \rho_{M}\right)$ is a right $D$-comodule if $M$ is an object in $(\mathcal{C}, c)$ and $\rho_{M}: M \rightarrow M \otimes D$ is a morphism in $\mathcal{C}$ satisfying $\left(M \otimes \varepsilon_{D}\right) \circ \rho_{M}=\operatorname{id}_{M},\left(\rho_{M} \otimes D\right) \circ \rho_{M}=\left(M \otimes \delta_{D}\right) \circ \rho_{M}$. Given two right $D$-comodules $\left(M, \rho_{M}\right)$ and $\left(N, \rho_{N}\right), f: M \rightarrow N$ is a morphism of right $D$-comodules if $\rho_{N} \circ f=(f \otimes D) \circ \rho_{M}$. We denote the category of right $D$-comodules by $\mathcal{C}^{D}$. Analogously, ${ }^{D} \mathcal{C}$ denotes the category of left $D$-comodules.

A bialgebra in $\mathcal{C}$ is an object $H$ with algebra and coalgebra structures, and such that $\varepsilon_{H}$ and $\delta_{H}$ are algebra morphisms (equivalently $\eta_{H}$ and $\mu_{H}$ are coalgebra morphisms). We say that $H$ is a Hopf algebra if there exists a morphism $\lambda_{H}: H \rightarrow H$ which is convolution inverse to the identical map. We call $\lambda_{H}$ the antipode of $H$.

If $H$ and $B$ are bialgebras $f: H \rightarrow B$ is a morphism of bialgebras if $f$ is a morphism of algebras and coalgebras. Moreover, in this case if $H$ and $B$ are Hopf algebras, is not difficult to see that $\lambda_{B} \circ f=f \circ \lambda_{H}$ and then we will say that $f$ is a morphism of Hopf algebras.

Let $H$ be a Hopf algebra in $\mathcal{C}$. Let $M$ be an algebra (a coalgebra) such that $\left(M, \psi_{M}\right)$ is in $\mathcal{C}_{H}$ (resp. $\left(M, \varphi_{M}\right)$ is in $\left.{ }_{H} \mathcal{C}\right)$. We say that $M$ is a right (resp. left) $H$-module (co)algebra if $\eta_{M}$ and $\mu_{M}\left(\varepsilon_{M}\right.$ and $\left.\delta_{M}\right)$ are morphisms of right (resp. letf) $H$-modules. If $\left(M, \rho_{M}\right)$ is in $\mathcal{C}^{H}$ (resp. $\left(M, r_{M}\right)$ is in $\left.{ }^{H} \mathcal{C}\right)$. We say that $M$ is a right (resp. left) $H$-comodule (co)algebra if $\eta_{M}$ and $\mu_{M}\left(\varepsilon_{M}\right.$ and $\left.\delta_{M}\right)$ are morphisms of right (resp. letf) $H$-comodules.

We say that a right $H$-module coalgebra $\left(M, \psi_{M}\right)$ is $H$-Cleft if there exists a cointegral $g$, i.e., an $H$-module morphism in $\operatorname{Reg}(M, H)$. Replacing $g$ by $\left(\left(\varepsilon_{H} \circ g^{-1}\right) \otimes g\right) \circ \delta_{M}$ we may assume that $\varepsilon_{H} \circ g=\varepsilon_{M}$. In this case, we will say that $g$ is a total cointegral. Analogously, a right $H$-comodule algebra $\left(M, \rho_{M}\right)$ is $H$-Cleft if there exists an integral $f$, i.e., an $H$-comodule morphism in $\operatorname{Reg}(H, M)$. Replacing $f$ by $\mu_{M} \circ\left(\left(f^{-1} \circ \eta_{H}\right) \otimes f\right)$ we may assume that $f \circ \eta_{H}=\eta_{M}$. In this case, we will say that $f$ is a total integral. 
Let $H$ be a bialgebra in $\mathcal{C},\left(A ; \varphi_{A}\right)$ be a left $H$-module algebra. We define

$$
\begin{aligned}
& \eta_{A \sharp H}=\eta_{A} \otimes \eta_{H}, \\
& \mu_{A \sharp H}=\left(\mu_{A} \otimes \mu_{H}\right) \circ\left(A \otimes \varphi_{A} \otimes H \otimes H\right) \circ\left(A \otimes H \otimes c_{H, A} \otimes H\right) \\
& \quad \circ\left(A \otimes \delta_{H} \otimes A \otimes H\right) .
\end{aligned}
$$

It is well know that $A \sharp H=\left(A \otimes H, \eta_{A \sharp H}, \mu_{A \sharp H}\right)$ is an algebra in $\mathcal{C}$, called the smash product.

On the other hand, if $\left(A, r_{A}\right)$ is a left $H$-comodule coalgebra, we have that $A \propto H=\left(A \otimes H, \varepsilon_{A \propto H}, \delta_{A \propto H}\right)$, where $\varepsilon_{A \propto H}=\varepsilon_{A} \otimes \varepsilon_{H}$ and $\delta_{A \propto H}=\left(A \otimes \mu_{H} \otimes A \otimes H\right) \circ\left(A \otimes H \otimes c_{A, H} \otimes H\right) \circ\left(A \otimes r_{A} \otimes H \otimes H\right) \circ\left(\delta_{A} \otimes \delta_{H}\right)$, is a coalgebra, called the smash coproduct.

\section{Two-cocycles and skew pairings}

Let $H$ be a bialgebra in $\mathcal{C}$. A morphism $\sigma \in \operatorname{Reg}(H \otimes H, K)$ is a two cocycle if

$$
\sigma \circ\left(H \otimes \mu_{\sigma H}\right)=\sigma \circ\left(\mu_{\sigma H} \otimes H\right)
$$

where $\mu_{\sigma H}=\left(\sigma \otimes \mu_{H}\right) \circ \delta_{H \otimes H}$.

It is well know (see Theorem 1.6.a of [5]) that if $\sigma$ is a two cocycle we have the next equalities:

a) $\left(\sigma \circ\left(\eta_{H} \otimes \eta_{H}\right)\right) *\left(\sigma^{-1} \circ\left(\eta_{H} \otimes \eta_{H}\right)\right)=\mathrm{id}_{K}$,

b) $\sigma \circ\left(H \otimes \eta_{H}\right)=\varepsilon_{H}=\sigma \circ\left(\eta_{H} \otimes H\right)$.

Moreover, if $H$ is a Hopf algebra $\sigma \circ\left(H \otimes \lambda_{H}\right) \in \operatorname{Reg}(H \otimes H, K)$ has inverse $\sigma^{-1} \circ\left(\lambda_{H} \otimes H\right)$.

Proposition 3.1. Let $H$ be a bialgebra in $\mathcal{C}$ and let $\sigma$ be a two cocycle in $\operatorname{Reg}(H \otimes H, K)$. Then:

a) The triple $\left(H, \eta_{H}, \mu_{\sigma H}\right)$ is an algebra in $\mathcal{C}$.

b) The triple $\left(H, \eta_{H}, \mu_{H_{\sigma-1}}\right)$ is an algebra in $\mathcal{C}$, where $\mu_{H_{\sigma-1}}=\left(\mu_{H} \otimes\right.$ $\left.\sigma^{-1}\right) \circ \delta_{H \otimes H}$.

Proof: We show b). The proof a) is analogous and we leave the details for the reader. Trivially, $\mu_{H_{\sigma-1}} \circ\left(\eta_{H} \otimes H\right)=\operatorname{id}_{H}=\mu_{H_{\sigma-1}} \circ\left(H \otimes \eta_{H}\right)$. Finally,

$$
\begin{aligned}
& \mu_{H_{\sigma^{-1}}} \circ\left(\mu_{H_{\sigma^{-1}}} \otimes H\right) \\
& =\mu_{H} \circ\left(H \otimes \mu_{H}\right) \circ\left(H \otimes H \otimes H \otimes\left(\sigma^{-1} \circ\left(\mu_{H_{\sigma^{-1}}} \otimes H\right)\right) \circ \delta_{H \otimes H \otimes H}\right. \\
& =\mu_{H} \circ\left(H \otimes \mu_{H}\right) \circ\left(H \otimes H \otimes H \otimes\left(\sigma^{-1} \circ\left(H \otimes \mu_{H_{\sigma^{-1}}}\right)\right) \circ \delta_{H \otimes H \otimes H}\right. \\
& =\mu_{H_{\sigma^{-1}}} \circ\left(H \otimes \mu_{H_{\sigma^{-1}}}\right) .
\end{aligned}
$$


Proposition 3.2. Let $H$ be a Hopf algebra in $\mathcal{C}$ and let $\sigma \in \operatorname{Reg}(H \otimes H, K)$ be a two cocycle. Then

$$
H^{\sigma}=\left(H, \eta_{H}, \mu_{(\sigma H)_{\sigma^{-1}}}, \varepsilon_{H}, \delta_{H}, \lambda_{H^{\sigma}}\right)
$$

is a Hopf algebra in $\mathcal{C}$ where $\lambda_{H^{\sigma}}=\left(f \otimes \lambda_{H} \otimes f^{-1}\right) \circ\left(H \otimes \delta_{H}\right) \circ \delta_{H}$ and $f=\sigma \circ\left(H \otimes \lambda_{H}\right) \circ \delta_{H}$ is a morphism in $\operatorname{Reg}(H, K)$ with inverse $f^{-1}=\sigma^{-1} \circ\left(\lambda_{H} \otimes H\right) \circ \delta_{H}$.

Proof: See $1.6(\mathrm{~b})$ of $[\mathbf{5}]$.

Let $B$ and $H$ be bialgebras in $\mathcal{C}$. A morphism $\tau: B \otimes H \rightarrow K$ is called a skew pairing on $(B, H)$ if:

a) $\tau \circ\left(\mu_{B} \otimes H\right)=(\tau \otimes \tau) \circ\left(B \otimes c_{B, H} \otimes H\right) \circ\left(B \otimes B \otimes \delta_{H}\right)$,

b) $\tau \circ\left(B \otimes \mu_{H}\right)=(\tau \otimes \tau) \circ\left(B \otimes c_{B, H} \otimes H\right) \circ\left(\delta_{B} \otimes c_{H, H}\right)$.

As a direct consequence of a) and b), if $\tau$ is a convolution invertible skew pairing on $(B, H)$, then $\tau \circ\left(\eta_{B} \otimes H\right)=\varepsilon_{H}$ and $\tau \circ\left(B \otimes \eta_{H}\right)=\varepsilon_{B}$.

Let $\tau$ be a convolution invertible skew pairing on $(B, H)$. Then the morphism $\sigma_{\tau}=\varepsilon_{B} \otimes\left(\tau \circ c_{H, B}\right) \otimes \varepsilon_{H}$ is a two cocycle in $\operatorname{Reg}(B \otimes H \otimes$ $B \otimes H, K)$, with inverse $\sigma_{\tau}^{-1}=\varepsilon_{B} \otimes\left(\tau^{-1} \circ c_{H, B}\right) \otimes \varepsilon_{H}$, called the two cocycle associated with $\tau$ (1.5 of $[\mathbf{6}])$.

Let $H, B$ be Hopf algebras in $\mathcal{C}$ and let $\tau$ be a convolution invertible skew pairing on $(B, H)$. Then $\left(B, \varphi_{B}\right)$ is a left $H$-module coalgebra and $\left(H, \psi_{H}\right)$ is a right $B$-module coalgebra, where

$$
\begin{aligned}
& \varphi_{B}=\left(\tau \otimes B \otimes \tau^{-1}\right) \circ\left(B \otimes H \otimes \delta_{B} \otimes H\right) \circ \delta_{B \otimes H} \circ c_{H, B}, \\
& \psi_{H}=\left(\left(\tau \circ c_{H, B}\right) \otimes H \otimes\left(\tau^{-1} \circ c_{H, B}\right)\right) \circ\left(H \otimes B \otimes \delta_{H} \otimes B\right) \circ \delta_{H \otimes B} .
\end{aligned}
$$

The object $B \bowtie_{\tau} H=\left(B \otimes H, \eta_{B \otimes H}, \mu_{B \bowtie_{\tau} H}, \varepsilon_{B \otimes H}, \delta_{B \otimes H}, \lambda_{B \bowtie_{\tau} H}\right)$ is a Hopf algebra in $\mathcal{C}$ where

$$
\begin{aligned}
& \mu_{B \bowtie_{\tau} H}=\left(\mu_{B} \otimes \mu_{H}\right) \circ\left(B \otimes \varphi_{B} \otimes \psi_{H} \otimes H\right) \circ\left(B \otimes \delta_{H \otimes B} \otimes H\right), \\
& \lambda_{B \bowtie_{\tau} H}=\left(\varphi_{B} \otimes \psi_{H}\right) \circ \delta_{H \otimes B} \circ\left(\lambda_{H} \otimes \lambda_{B}\right) \circ c_{B, H} .
\end{aligned}
$$

Moreover, if $\sigma_{\tau}$ is the two cocycle associated with $\tau$ then the Hopf algebras $(B \otimes H)^{\sigma_{\tau}}$ and $B \bowtie_{\tau} H$ are the same [6] and [4].

\section{The Hopf algebras $B \bowtie_{\tau} H$ and $B \infty H$}

A quasitriangular Hopf algebra in $\mathcal{C}$ is a pair $(H, \mathcal{R})$ where $H$ is a Hopf algebra in $\mathcal{C}$ and $\mathcal{R}$ is a morphism in $\operatorname{Reg}(K, H \otimes H)$ such that:

a) $\left(\delta_{H} \otimes H\right) \circ \mathcal{R}=\left(H \otimes H \otimes \mu_{H}\right) \circ\left(H \otimes c_{H, H} \otimes H\right) \circ(\mathcal{R} \otimes \mathcal{R})$.

b) $\left(H \otimes \delta_{H}\right) \circ \mathcal{R}=\left(\mu_{H} \otimes c_{H, H}\right) \circ\left(H \otimes c_{H, H} \otimes H\right) \circ(\mathcal{R} \otimes \mathcal{R})$. 
c) $\mu_{H \otimes H} \circ\left(\left(c_{H, H} \circ \delta_{H}\right) \otimes \mathcal{R}\right)=\mu_{H \otimes H} \circ\left(\mathcal{R} \otimes \delta_{H}\right)$.

If $(H, \mathcal{R})$ is a quasitriangular Hopf algebra in $\mathcal{C}$, it is not difficult to prove that the morphism $\mathcal{R}$ obeys:

1) $\left(\varepsilon_{H} \otimes H\right) \circ \mathcal{R}=\eta_{H}=\left(H \otimes \varepsilon_{H}\right) \circ \mathcal{R}$

2) $\left(\mu_{H} \otimes \mu_{H} \otimes \mu_{H}\right) \circ\left(H \otimes c_{H, H} \otimes c_{H, H} \otimes H\right) \circ(\mathcal{R} \otimes \mathcal{R} \otimes \mathcal{R})$

$$
\begin{gathered}
=\left(\mu_{H} \otimes H \otimes\left(\mu_{H} \circ c_{H, H}\right)\right) \circ\left(H \otimes H \otimes c_{H, H} \otimes H\right) \\
\quad \circ\left(H \otimes c_{H, H} \otimes\left(\mu_{H} \circ c_{H, H}\right) \otimes H\right) \circ(\mathcal{R} \otimes \mathcal{R} \otimes \mathcal{R})
\end{gathered}
$$

3) $\mathcal{R}^{-1}=\left(\lambda_{H} \otimes H\right) \circ \mathcal{R}$

4) $\left(\lambda_{H} \otimes \lambda_{H}\right) \circ \mathcal{R}=\mathcal{R}$

5) $\left(H \otimes \lambda_{H}\right) \circ \mathcal{R}^{-1}=\mathcal{R}$

6) $\left(\mu_{H \otimes H} \otimes H\right) \circ\left(H \otimes H \otimes \delta_{H} \otimes H\right) \circ(\mathcal{R} \otimes \mathcal{R})$

$$
=\left(H \otimes \mu_{H \otimes H}\right) \circ\left(H \otimes \mathcal{R} \otimes \delta_{H}\right) \circ \mathcal{R} .
$$

Let $H$ be a Hopf algebra in $(\mathcal{C}, c)$. Let $\left(M, \varphi_{M}\right)$ be in ${ }_{H} \mathcal{C}$ and $\left(M, r_{M}\right)$ be in ${ }^{H} \mathcal{C}$. We say that $\left(M, \varphi_{M}, r_{M}\right)$ is in ${ }_{H}^{H} \mathcal{Y D}$ if $\varphi_{M}$ and $r_{M}$ satisfies the compatibility condition:

$$
\begin{aligned}
\left(\mu_{H} \otimes M\right) & \circ\left(H \otimes c_{M, H}\right) \circ\left(\left(r_{M} \circ \varphi_{M}\right) \otimes H\right) \circ\left(H \otimes c_{H, M}\right) \\
& \circ\left(\delta_{H} \otimes M\right)=\left(\mu_{H} \otimes \varphi_{M}\right) \circ\left(H \otimes c_{H, H} \otimes M\right) \circ\left(\delta_{H} \otimes r_{M}\right) .
\end{aligned}
$$

If $B$ is a Hopf algebra in ${ }_{H}^{H} \mathcal{Y D}$ with antipode $\lambda_{B}$ then

$$
B \propto H=\left(B \otimes H, \eta_{B \sharp H}, \mu_{B \sharp H}, \varepsilon_{B \propto H}, \delta_{B \propto H}\right)
$$

is a Hopf algebra in $\mathcal{C}$ with antipode

$$
\begin{aligned}
& \lambda_{B \infty H}=\left(\varphi_{B} \otimes H\right) \circ\left(H \otimes c_{H, B}\right) \circ\left(\delta_{H} \otimes B\right) \circ\left(\lambda_{H} \otimes \lambda_{B}\right) \\
& \circ\left(\mu_{H} \otimes B\right) \circ\left(H \otimes c_{B, H}\right) \circ\left(r_{B} \otimes H\right)
\end{aligned}
$$

(see $[\mathbf{2}]$ for more details).

Theorem 4.1. Let $B$ and $H$ be Hopf algebras in $\mathcal{C}$. Assume that $H$ is quasitriangular. Let $\tau \in \operatorname{Reg}(B \otimes H, K)$ be a skew pairing on $(B, H)$. Then $B$ is a Hopf algebra in the category ${ }_{H}^{H} \mathcal{Y D}$ and there exists a Hopf algebra isomorphism $w: B \infty H \rightarrow B \bowtie_{\tau} H$.

Proof: By 2.5 of $[\mathbf{6}], g: B \bowtie_{\tau} H \rightarrow H$, defined by

$$
g=\left(\tau \otimes\left(\mu_{H} \circ c_{H, H}\right)\right) \circ\left(B \otimes c_{H, H} \otimes H\right) \circ(B \otimes H \otimes \mathcal{R})
$$

and $f=\eta_{B} \otimes H: H \rightarrow B \bowtie_{\tau} H$ are Hopf algebra morphisms such that $g \circ f=\operatorname{id}_{H}$. Then $\left(B \bowtie_{\tau} H, \rho_{B \bowtie_{\tau} H}\right)$ where

$\rho_{B \bowtie_{\tau} H}=\left(B \otimes H \otimes \tau \otimes\left(\mu_{H} \circ c_{H, H}\right)\right) \circ\left(B \otimes H \otimes B \otimes c_{H, H} \otimes H\right) \circ\left(\delta_{B \otimes H} \otimes \mathcal{R}\right)$ 
is a right $H$-Cleft comodule algebra with total integral $f$ and $\left(B \bowtie_{\tau} H\right.$, $\left.\psi_{B \bowtie_{\tau} H}=B \otimes \mu_{H}\right)$ is a right $H$-Cleft module coalgebra with total cointegral $g$. Thus, applying 3.2 of $[\mathbf{1}]$, the object $\left(B \bowtie_{\tau} H\right)_{0}$ defined by the equalizer diagram

$$
\left(B \bowtie_{\tau} H\right)_{0} \stackrel{i_{B \bowtie_{\tau} H}}{\longrightarrow} B \bowtie_{\tau} H \underset{B \bowtie_{\tau} H \otimes \eta_{H}}{\stackrel{\rho_{B \bowtie_{\tau} H}}{\longrightarrow}} B \bowtie_{\tau} H \otimes H
$$

is a Hopf algebra in ${ }_{H}^{H} \mathcal{Y D}$ such that $\left(B \bowtie_{\tau} H\right)_{0} \infty H$ and $B \bowtie_{\tau} H$ are isomorphic Hopf algebras. Moreover, $\left(B \bowtie_{\tau} H\right)^{0}$ defined by the coequalizer of $\psi_{B \bowtie_{\tau} H}$ and $B \otimes H \otimes \varepsilon_{H}$ is such that $\left(B \bowtie_{\tau} H\right)^{0}=\left(B \bowtie_{\tau} H\right)_{0}$ and therefore $\left(B \bowtie_{\tau} H\right)^{0}=\left(B \bowtie_{\tau} H\right)_{0}=B$ because the coequalizer morphism of $\psi_{B \bowtie_{\tau} H}$ and $B \otimes H \otimes \varepsilon_{H}$ is $p=B \otimes \varepsilon_{H}$. As a consequence, $\left(B, \eta_{B}, m_{B}, \varepsilon_{B}, \delta_{B}, s_{B}\right)$ where $m_{B}=\mu_{B} \circ\left(B \otimes \varphi_{B}\right) \circ\left(i_{B \bowtie_{\tau} H} \otimes B\right)$ and $s_{B}=\left(\tau \otimes \varphi_{B}\right) \circ\left(B \otimes \mathcal{R} \otimes \lambda_{B}\right) \circ \delta_{B}$ is a Hopf algebra in ${ }_{H}^{H} \mathcal{Y} \mathcal{D}$.

The isomorphism $w$ between $B \infty H$ and $B \bowtie_{\tau} H$ is $w=\mu_{B \bowtie_{\tau} H} \circ$ $\left(i_{B \bowtie_{\tau} H} \otimes f\right)$. Note that $w=\left(B \otimes \tau \otimes \mu_{H}\right) \circ\left(\delta_{B} \otimes H \otimes \lambda_{H} \otimes H\right) \circ(B \otimes \mathcal{R} \otimes H)$ because

$$
\begin{aligned}
i_{B \bowtie_{\tau} H} & =i_{B \bowtie_{\tau} H} \circ p \circ\left(B \otimes \eta_{H}\right) \\
& =\mu_{B \bowtie_{\tau} H} \circ\left(B \bowtie_{\tau} H \otimes f^{-1}\right) \circ \rho_{B \bowtie_{\tau} H} \circ\left(B \otimes \eta_{H}\right) \\
& =\mu_{B \bowtie_{\tau} H} \circ\left(B \otimes H \otimes \eta_{B} \otimes \lambda_{H}\right) \circ \rho_{B \bowtie_{\tau} H} \circ\left(B \otimes \eta_{H}\right) \\
& =\left(B \otimes \tau \otimes \lambda_{H}\right) \circ\left(\delta_{B} \otimes \mathcal{R}\right) .
\end{aligned}
$$

Finally, we compute $\varphi_{\left(B \bowtie_{\tau} H\right)_{0}}$ and $r_{\left(B \bowtie_{\tau} H\right)_{0}}$,

$$
\begin{aligned}
& i_{B \bowtie_{\tau} H} \circ \varphi_{\left(B \bowtie_{\tau} H\right)_{0}} \\
& =\mu_{B \bowtie_{\tau} H} \circ\left(B \otimes H \otimes\left(\mu_{B \bowtie_{\tau} H} \circ c_{B \otimes H, B \otimes H}\right)\right) \\
& =\left(B \otimes \mu_{H}\right) \circ\left(\varphi_{B} \otimes \psi_{H} \otimes \tau \otimes \mu_{H}\right) \circ\left(\delta_{H \otimes B} \otimes B \otimes H \otimes f^{-1} \otimes i_{B \bowtie_{\tau} H}\right) \circ\left(\delta_{H} \otimes B\right) \\
& \quad \circ\left(H \otimes \delta_{B} \otimes \mathcal{R} \otimes H\right) \circ\left(H \otimes c_{H, B}\right) \otimes\left(H \otimes \lambda_{H} \otimes B\right) \circ\left(\delta_{H} \otimes B\right) \\
& =\left(B \otimes \mu_{H}\right) \circ\left(\varphi_{B} \otimes \psi_{H} \otimes \tau \otimes H\right) \circ\left(\delta_{H \otimes B} \otimes B \otimes H \otimes\left(\lambda_{H} \circ \mu_{H} \circ c_{H, H}\right)\right) \\
& =i_{B \bowtie_{\tau} H} \circ \varphi_{B} . \quad \circ\left(H \otimes \delta_{B} \otimes \mathcal{R} \otimes H\right) \circ\left(H \otimes c_{H, B}\right) \circ\left(\delta_{H} \otimes B\right)
\end{aligned}
$$

Thus, $\varphi_{\left(B \bowtie_{\tau} H\right)_{0}}=p \circ i_{B \bowtie_{\tau} H} \circ \varphi_{\left(B \bowtie_{\tau} H\right)_{0}}=p \circ i_{B \bowtie_{\tau} H} \circ \varphi_{B}=\varphi_{B}$ and, as a consequence, the left $H$-module coalgebra $\left(B, \varphi_{B}\right)$ is a left $H$-module algebra too. 
On the other hand,

$$
\begin{aligned}
& r\left(B \bowtie_{\tau} H\right)_{0} \\
& =(g \otimes p) \circ \delta_{B \otimes H} \circ i_{B \bowtie_{\tau} H} \\
& =\left(\tau \otimes c_{B, H}\right) \circ\left(B \otimes H \otimes B \otimes \tau \otimes \mu_{H}\right) \circ\left(B \otimes H \otimes \delta_{B} \otimes c_{H, H} \otimes \lambda_{H}\right) \\
& \circ\left(B \otimes c_{B, H} \otimes H \otimes H \otimes H\right) \circ\left(\delta_{B} \otimes \mathcal{R} \otimes \mathcal{R}\right) .
\end{aligned}
$$

Acknowledgements. The authors has been supported by the Xunta de Galicia, Project: PGIDT00PXI20706PR, and by the Ministerio de Ciencia y Tecnología, Project: BFM2000-0513-C02-02.

\section{References}

[1] J. N. Alonso Alvarez and J. M. Fernández Vilaboa, Cleft extensions in braided categories, Comm. Algebra 28(7) (2000), $3185-3196$.

[2] Y. Bespalov, Crossed modules and quantum groups in braided categories, Appl. Categ. Structures 5(2) (1997), 155-204.

[3] S. Caenepeel, S. Dăscălescu, G. Militaru and F. Panaite, Coalgebra deformations of bialgebras by Harrison cocycles, copairings of Hopf algebras and double crosscoproducts, Bull. Belg. Math. Soc. Simon Stevin 4(5) (1997), 647-671.

[4] Hui-Xiang Chen, Braiding structures of double crossproducts, Tsukuba J. Math 22(3) (1998), 589-609.

[5] Y. DoI, Braided bialgebras and quadratic bialgebras, Comm. Algebra 21(5) (1993), 1731-1749.

[6] Y. DoI AND M. TAKeuchi, Multiplication alteration by two cocycles - the quantum version. Comm. Algebra 22(14) (1994), $5715-5732$.

[7] S. Mac Lane, "Categories for the working mathematicien", Graduate Texts in Mathematics 5, Springer-Verlag, New York-Berlin, 1971.

[8] S. MAJID, Braided matrix structure of the Sklyanin algebra and of the quantum Lorentz group, Comm. Math. Phys. 156(3) (1993), 607-638.

[9] S. MAJID, "Foundations of quantum group theory", Cambridge University Press, Cambridge, 1995.

[10] D. RADFORD, The structure of Hopf algebras with a projection, J. Algebra 92(2) (1985), 322-347. 
J. N. Alonso Alvarez:

Departamento de Matemáticas

Universidad de Vigo

Campus Universitario Lagoas-Marcosende

E-36280, Vigo

Spain

E-mail address: jnalonso@uvigo.es

J. M. Fernández Vilaboa:

Departamento de Alxebra

Universidad de Santiago de Compostela

E-15771, Santiago de Compostela

Spain

E-mail address: vilaboa@zmat.usc.es

R. González Rodríguez:

Departamento de Matemática Aplicada

Universidad de Vigo

Campus Universitario Lagoas-Marcosende

E-36280, Vigo

Spain

E-mail address: rgon@dma.uvigo.es

Primera versió rebuda el 3 de novembre de 2000, darrera versió rebuda el 3 de juliol de 2001. 